\title{
Night Call in a Teaching Hospital: 1979 and 2019
}

\author{
Shaurya Taran, MD' ${ }^{1}$ Benjamin Chin-Yee, MD, MA ${ }^{1}$, Allan S Detsky MD, PhD ${ }^{2,3^{*}}$
}

'Department of Medicine, University of Toronto, Toronto, Ontario, Canada; ${ }^{2}$ Institute for Health Policy, Management, and Evaluation, and Department of Medicine, University of Toronto, Toronto, Ontario, Canada; ${ }^{3}$ Department of Medicine, Mount Sinai Hospital and University Health Network, Toronto, Ontario, Canada.

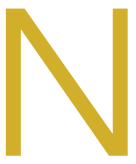

o matter the era, few aspects of residency are more defining or memorable than overnight call. Nights can be a time of growth and learning but also of fear and uncertainty, as residents take on the responsibility of managing sick patients on their own. One of us (ASD) started his residency in 1978 at the Massachusetts General Hospital in Boston; the other two (ST and BCY) started theirs in 2016 and 2017, respectively, at the University of Toronto. In this essay, we reflect on our experiences of night call separated by 40 years, highlighting what has changed and what has stayed the same.

\section{9}

At 6 PM, a calm overtook the hospital as the daytime staff emptied out of the building. The only remaining residents were those on call for the night. Each team had one resident and one medical student responsible for about 20 inpatients. Teams also admitted new patients from the emergency department (ED). Since there were three teams who rotated the admissions, we were able to spend a part of our night on the inpatient ward. Most of the new patients had been thoroughly evaluated by a medical resident in the ED before we saw them, giving us a head start on eliciting their stories, performing physical examinations, reviewing the laboratory workup, and generating plans to present to the attending physician (who was called "the visit") the next morning at $10 \mathrm{Am}$.

We carried one pager that was about 7 inches long and 2 inches wide clipped to the waist of our pants. It could only make a beep; we then had to call the page operator to find out who wanted us. However, the pages were relatively few. Nurses called only when a patient was unstable, and other residents called only when a new patient was ready in the emergency department. At 9 PM, the laboratory data for the day were delivered to the ward nursing stations on computer-generated paper. Our job was to separate the pages connected by serrated breaks, review the results, and then file the pages in the charts. The nurses were aware of this routine, so they saved their questions for our presence on the wards, reducing the need to page us. At 10 PM, residents from all services went to

*Corresponding Author: Allan S. Detsky, MD, , PhD; E-mail: allan.detsky@ sinaihealthsystem.ca; Telephone: 416-586-8507

Published online first August 21, 2019.

Received: April 25, 2019; Revised: June 26, 2019; Accepted: July 7, 2019

๑ 2019 Society of Hospital Medicine DOI 10.12788/jhm.3284 the cafeteria for the "10 o'clock meal" when the food was free. We learned early in the year to drop everything at 10 PM; otherwise, we did not eat. The social aspect of the group meal was comforting, but compared with today, the group was much more homogeneous and therefore less interesting. There were several women medical residents (although almost none in surgery), but very few minorities, and no openly gay residents.

Gathering data about patients prior to the current hospitalization required reviewing the "old chart," which had to be delivered from patient records but was generally available when the patient was still in the ED. It contained typed discharge summaries and progress notes often handwritten by coresidents whom we knew. The handwriting was often difficult to read, outpatient notes were not included, and information from other hospitals was absent-but despite these deficiencies, we somehow managed just fine.

The patients on the inpatient ward were mostly stable, but more importantly, we had very few medications and tests to order. I recall prescribing fewer than 20 drugs-furosemide, hydrochlorothiazide, penicillins, cephalosporins, gentamicin, isoniazid, lidocaine, nitroglycerin, aminophylline, alpha-methyldopa, clonidine, propranolol, digoxin, hydralazine, indomethacin, steroids, and morphine. Orders for tests and imaging had to be physically written in the chart and could not be inputted remotely, which was a nuisance when we were away from the ward. However, we rarely ordered any imaging beyond plain radiographs at night. We did draw arterial blood gases and venous blood, administer oxygen, insert intravenous and central lines, take electrocardiograms, and perform urinalyses by microscopy. We did all these tasks ourselves for patients on the "ward service" (as opposed to the "private service", which had to do with the type of insurance the patients possessed). As a result, we became experts in both blood drawing and intravenous line insertion - skills that might be less familiar to today's residents.

Of course, patients did get acutely ill during the night. I recall intubating, cardioverting, performing phlebotomy to alleviate pulmonary edema, sending patients to surgery, and pronouncing death. Nevertheless, we often got sleep, and sometimes, several hours in a row. I had a rule; I always took a shower the next morning and put on clean clothes (we stayed until 5 PM, making the shifts 33.5 hours long). There was a camaraderie that existed between all of us at night. We were supportive, friendly, and knew each other by name, and more.

We were often frightened by the responsibility of managing sick patients alone. On particularly challenging nights, we would record our fears and feelings in a "night call diary" in 
one of the conference rooms-generally at 4 AM. Some entries became legendary as people read and reread those months and years later. Mornings always brought a sense of relief and accomplishment, because when the sun came up, we knew that the other residents would not be far behind; when they arrived, we could tell our stories and get help.

There was definitely competitiveness to the work. Those who responded quickly to deteriorating patients were applauded; those who did not really know what to do were subtly disdained. However, over time, we all got the hang of it, and this led to a growing confidence that we were indeed doctors. The graded autonomy afforded by night call was a crucial part of that journey.

\section{9}

At 6 PM, the on-call residents assemble in the ED, where we would spend the remainder of the night and early morning admitting new patients to the hospital. A night team consists of a senior resident, three junior residents, and two medical students, with each resident being responsible for approximately 20 inpatients. Overnight coverage of the ward mostly occurs remotely; since the ED is often so busy, we address most of the issues through a computer or over the phone. Only in rare cases, such as when a patient is unstable or a death has occurred, do we deal with the matter in person.

To enable rapid remote responses, we each carry an assortment of devices on our waists or lanyards and in our pockets, such as a personal pager, ED consult pager, code blue pager, and hospital-issued smartphones capable of receiving pages, text messages, phone calls, and e-mails. Nurses, pharmacists, and other consultants communicate with us through all of these channels. Few of these interactions occur face-to-face. To our frustration, encounters with patients are frequently interrupted by a stream of beeps, rings, and vibrations-irrespective of whether we are having a difficult discussion about goals of care or performing a delicate procedure.

The ED contains a work space dedicated for residents to enter electronic orders, type notes, and review new admissions. Between consults, we try to discuss exciting cases and provide teaching to the medical students and interns, which we enjoy. Dinner is generally devoured while inputting orders. In exceptional circumstances, a brief reprieve from pages may allow the on-call team to share a meal. Depending on our role, sleep may be possible on certain nights but is never guaranteed. Moments spent with the on-call team-all of us learning, commiserating, and growing together-are some of the most memorable of residency, and many of us become close friends by the end of the rotation.

However, apart from these few familiar faces, we rarely get acquainted with the nurses or residents from other services. Many often refer to themselves by specialty rather than name and phone calls that begin with "Are you Medicine?" can end with "You should really call Orthopedics." Meanwhile, "Medicine" and "Orthopedics" may pass each other in the hallway without recognition beyond a vague familiarity of a voice heard on the phone.
Every 10 minutes spent with a new patient is accompanied by approximately one hour of "electronic" time, which includes reading through previous medical records, reviewing laboratory data and imaging, and creating an admission note. Interns might groan as they pull up a patient's electronic health record (EHR); irrelevant details often arise with each click of the mouse, and the cursed "copy-paste" function means that new notes often duplicate older ones. However, with time, we learn to look past the EHR's shortcomings and appreciate several of its advantages. For example, we are now able to access test results performed outside our hospital and thus limit our repetition of investigations. We can also use the EHR to rapidly glean salient information about a patient in time-critical scenarios. This is always a satisfying process, and it makes us wonder how physicians ever practiced in the era before computers.

Today's patients are older and sicker than ever before. Many are receiving treatments that did not exist even a decade ago. As residents, we must recognize a seemingly endless variety of drugs - a challenging but intellectually satisfying responsibility. We must also decide whether the patient's current health state permits their continuation, or whether safer alternatives exist. Some of these decisions cannot wait until the morning.

During handover at $8 \mathrm{AM}$, we often recount moments from our call shift with a sense of vigor that is only partly dulled by fatigue. We may share with pride our management of a sick patient. We may relay a touching exchange with a concerned family member. Or we may recall with satisfaction our handling of a tense situation with a colleague. Taking part in these experiences is one of the most character-building aspects of being on call. The absence of our supervisors can be unnerving at first, but we gradually begin to enjoy the sense of independence. Moreover, we feel empowered to make our patients better with the right combination of carefully selected treatments. Nothing makes us feel more like doctors.

No doubt, being on call is difficult. The next day brings a feeling of relief and accomplishment, knowing that we got through it-whether by floundering or flourishing -in one piece.

\section{CONCLUSION}

The two passages described here are personal descriptions of a typical night on-call in two different eras. Readers around the world may have a very different recollection of their own experience. Nevertheless, several aspects of being on call remain constant, such as anxiety about caring for sick patients alone, fond recollections of friends made, and relief when the morning comes. Most important, however, might be the tremendous satisfaction at the opportunity to learn and grow-to become a competent physician by testing one's physical and intellectual limits through graded autonomy

On the other hand, certain elements of night call have undeniably changed-partly a consequence of the increased number of people involved in patient care and changing communication technology. Residents today encounter a greater number of interruptions to their work flow. Tasks that require long, continuous periods of full attention are now punctuated by texts, 
e-mails, calls, and pages. The EHR is often clumsy to navigate, but it can also be a veritable mine of information. Finally, although residents from the same specialty may be close friends, duty hour restrictions and remote asynchronous communication may reduce familiarity with residents from other programs.

Do these descriptions resonate with your experience of night call? Keeping in mind that the 1979 vignette is described through the rose-colored lens of nostalgia, both eras have their advantages and disadvantages. We leave it to the reader to decide what has changed (plus ça change) and what has stayed the same (plus c'est la même chose).

\section{Acknowledgments}

The authors thank Micheal A. Fifer, MD (Massachusetts General Hospital), and Timothy J. Judson, MD (UCSF), for their comments on an earlier draft of this essay.

Disclosures: The authors have nothing to disclose. 